\title{
INVARIANCE OF DOMAIN THEOREMS FOR CONDENSING MULTIVALUED VECTOR FIELDS
}

\author{
IN-SoOK KIM
}

\begin{abstract}
Using a degree theory for countably condensing multivalued maps, we show that under certain conditions an invariance of domain theorem holds for countably condensing or countably $k$-contractive multivalued vector fields.
\end{abstract}

\section{Introduction}

The celebrated invariance of domain theorem can be formulated as follows (see [10]):

Let $\Omega$ be a domain in a Banach space $X$ and $f: \Omega \rightarrow X$ a completely continuous map. If the vector field $I-f$ is injective, then $(I-f)(\Omega)$ is a domain in $X$. Here I denotes the identity map.

It is known that this theorem is closely related to the problem of finding solutions for nonlinear equations; see [11]. It is natural to investigate this problem for a large class of condensing maps in a more general setting. We focus our attention on the class of countably condensing maps, roughly speaking, condensing on countable subsets of the space. The use of such countable sets to solve nonlinear differential equations has been developed by Mönch [8].

Applying homotopy theory, Hahn [3] obtained the invariance of domain theorem for $k$-contractive maps which was generalized to a class of countably $k$-contractive maps in [5], whereas it was studied in $[2,6]$ based upon degree theory. Introducing degree theory for compact multivalued vector fields, Ma [7] established this theorem for these vector fields which was extended to condensing or $k$-contractive vector fields in [9], where the degree for ultimately compact multivalued maps is defined by means of Ma's degree.

The purpose of this paper is to study the above problem for a large class of countably condensing multivalued maps. The method is to use degree theory

Received October 29, 2008; Revised December 12, 2008.

2000 Mathematics Subject Classification. 47H09, 54C60, 47H11.

Key words and phrases. invariance of domain, countably condensing, multivalued vector fields, degree theory. 
for countably condensing multivalued maps due to Väth $[12,13]$. Firstly, we establish an invariance of domain theorem for countably condensing multivalued vector fields under the locally nonopposite condition. Secondly, we show that an invariance of domain theorem holds for countably $k$-contractive multivalued vector fields under the local boundary condition, where a key tool is Borsuk's theorem for odd countably condensing multivalued maps. Consequently, the invariance of domain theorems presented here include a number of known results of Ma [7], Petryshyn and Fitzpatrick [9] for multivalued maps, as well as Schauder [10] and Hahn [3] for single-valued maps.

\section{Degree theory}

In this section, we introduce a degree theory for countably condensing multivalued maps which is based on the degree for compact multivalued vector fields by Ma [7]; see [4, 12-14].

In what follows, we will denote by $X$ a Fréchet space unless specified otherwise. For a nonempty subset $\Omega$ of $X$, the closure, the boundary, the convex hull, and the closed convex hull of $\Omega$ in $X$ are denoted by $\bar{\Omega}, \partial \Omega$, co $\Omega$, and $\overline{\mathrm{co}} \Omega$, respectively. Let $k(X)$ and $k c(X)$ denote the collection of all nonempty compact subsets of $X$ and of all nonempty compact convex subsets of $X$, respectively.

Definition 1. A function $\gamma: 2^{X} \rightarrow[0, \infty]$ is said to be a measure of noncompactness on $X$ provided that the following conditions hold for any sets $M, N \subset X$ and for any real number $t$ :

(1) $\gamma(\overline{\mathrm{co}} M)=\gamma(M)$;

(2) $\gamma(M)=0$ if and only if $M$ is precompact;

(3) $\gamma(M \cup N)=\max \{\gamma(M), \gamma(N)\}$;

(4) $\gamma(M+N) \leq \gamma(M)+\gamma(N)$; and

(5) $\gamma(t M)=|t| \gamma(M)$.

Example 1. Let $X$ be a Banach space. The Kuratowski measure of noncompactness, $\alpha: 2^{X} \rightarrow[0, \infty]$, is defined as follows: If $M \subset X$ is unbounded, then $\alpha(M):=\infty$; if $M$ is bounded, then

$\alpha(M):=\inf \{d>0: M$ admits a finite cover by sets of diameter less than $d\}$.

The Hausdorff measure of noncompactness, $\beta: 2^{X} \rightarrow[0, \infty]$, is defined as follows: If $M \subset X$ is unbounded, then $\beta(M):=\infty$; if $M \subset X$ is bounded, then

$\beta(M):=\inf \{\varepsilon>0: M$ can be covered by finitely many balls of radius $\varepsilon\}$.

Then $\alpha$ and $\beta$ satisfy the above conditions; see $[1,2]$.

Definition 2. Let $\Omega$ be a nonempty subset of $X$ and $\gamma$ a measure of noncompactness on $X$. An upper semicontinuous map $F: \Omega \rightarrow 2^{X}$ is said to be condensing (with respect to $\gamma$ ) if for each set $B \subset \Omega$ the inequality $\gamma(B) \leq \gamma(F(B)$ ) implies that $B$ is relatively compact. More generally, an upper semicontinuous map $F: \Omega \rightarrow 2^{X}$ is said to be countably condensing (with respect to $\gamma$ ) if for 
each countable set $C \subset \Omega$ the inequality $\gamma(C) \leq \gamma(F(C))$ implies that $C$ is relatively compact. An upper semicontinuous homotopy $H:[0,1] \times \Omega \rightarrow 2^{X}$ is said to be countably condensing (with respect to $\gamma$ ) if for each countable set $C \subset \Omega$ the inequality $\gamma(C) \leq \gamma(H([0,1] \times C))$ implies that $C$ is relatively compact.

The following result which enables us to define a degree for countably condensing maps is taken from [14, Corollary 2.1].

Proposition 1. Let $D$ be a nonempty closed subset of a Fréchet space $X$ and let $V$ and $K$ be two compact subsets of $X$. If $H:[0,1] \times D \rightarrow k(X)$ is a countably condensing map, then there exists a compact convex subset $S$ of $X$ such that

(1) $S$ contains $K$;

(2) $H([0,1] \times(D \cap S))+V$ is a subset of $S$; and

(3) for each $(t, x) \in[0,1] \times D$, the relation $x \in c o[(H(t, x)+V) \cup S]$ implies $x \in S$.

If we take $K=\left\{x_{0}\right\}$ for some $x_{0} \in D$, then $D \cap S$ is not empty. Such a closed convex nonempty set $S$ satisfying conditions (2) and (3) is called a fundamental set for $H$.

We need the following result for odd countably condensing maps. The proof of Proposition 2.3 in [6] for single-valued maps carries over to multivalued maps.

Proposition 2. Let $D$ be a symmetric closed set in $X$ with $0 \in D$. If $F: D \rightarrow$ $k(X)$ is an odd countably condensing map, then there is a symmetric, convex and compact subset $S$ of $X$ with $0 \in S$ such that $F(D \cap S)$ is a subset of $S$ and the relation $x \in \operatorname{co}(F x \cup S)$ implies $x \in S$.

One may define a topological degree for countably condensing multivalued vector fields by means of Ma's degree.

Definition 3. Let $\Omega$ be a nonempty open set in $X$ and $y$ an arbitrary point of $X$. Suppose that $F: \bar{\Omega} \rightarrow k c(X)$ is a countably condensing map such that $x \notin F x+y$ for all $x \in \partial \Omega$. In view of Proposition 1 , there exists a compact fundamental set $S$ for $F$ such that

$$
F(\bar{\Omega} \cap S)+y \subset S .
$$

Let $R: X \rightarrow S$ be any retraction onto $S$. Then $F \circ R$ is compact on the closure of $R^{-1}(\Omega)$ and $y$ does not belong to $(I-F \circ R)\left(\partial R^{-1}(\Omega)\right)$. Hence Ma's degree for the compact multivalued map $F \circ R, d_{M}\left(I-F \circ R, R^{-1}(\Omega), y\right)$, is defined. Now we define the degree of $I-F$ on $\Omega$ with respect to $y$

$$
\operatorname{deg}(I-F, \Omega, y):=d_{M}\left(I-F \circ R, R^{-1}(\Omega), y\right) .
$$

This definition does not depend on the choice of $S$ and $R$; see [9, Lemma 2.2].

The above degree has the usual properties which will often be used; see $[12$, $13]$. 
Lemma 1. Let $\Omega$ be a nonempty open subset of $X$. Then the following statements hold:

(1) (Existence) Let $F: \bar{\Omega} \rightarrow k c(X)$ be a countably condensing map and $y$ a point of $X$ such that $x \notin F x+y$ for all $x \in \partial \Omega$. If $\operatorname{deg}(I-F, \Omega, y) \neq 0$, then there exists a point $x$ in $\Omega$ such that $x \in F x+y$.

(2) (Normalization) If $x_{0} \in \Omega$ and $F x=\left\{x_{0}\right\}$ for all $x \in \bar{\Omega}$, then $\operatorname{deg}(I-$ $F, \Omega, 0)=1$.

(3) (Homotopy invariance) If $H:[0,1] \times \bar{\Omega} \rightarrow k c(X)$ is a countably condensing homotopy such that $x \notin H(t, x)$ for all $(t, x) \in[0,1] \times \partial \Omega$, then

$$
\operatorname{deg}(I-H(0, \cdot), \Omega, 0)=\operatorname{deg}(I-H(1, \cdot), \Omega, 0) .
$$

We close this section with the following result which is helpful for proving an invariance of domain theorem. The case of condensing or compact maps can be found in [9, Lemma 4.1] or [7, Corollary 7.8].

Lemma 2. Let $\Omega$ be a nonempty open subset of $X$. Suppose that $F: \bar{\Omega} \rightarrow$ $k c(X)$ is a countably condensing map with respect to $\gamma$ such that $x \notin F x$ for all $x \in \partial \Omega$. Then there exists a convex open neighborhood $V$ of the origin 0 in $X$ such that if $G: \bar{\Omega} \rightarrow k c(X)$ is a countably condensing map and $G x \subset F x+V$ for all $x \in \partial \Omega$, then

$$
\operatorname{deg}(I-G, \Omega, 0)=\operatorname{deg}(I-F, \Omega, 0) ;
$$

in particular, $\operatorname{deg}(I-F, \Omega, y)=\operatorname{deg}(I-F, \Omega, 0)$ for every $y \in V$.

Proof. We first claim that there is a convex open neighborhood $V$ of 0 in $X$ such that

$$
(x-F x) \cap V=\emptyset \text { for all } x \in \partial \Omega .
$$

Indeed, if this were not the case, then let $\left\{W_{n}: n \in \mathbb{N}\right\}$ be a monotonically decreasing basis of convex open neighborhoods of 0 in $X$ such that $x_{n} \in \partial \Omega, y_{n} \in$ $W_{n}$, and $z_{n} \in F x_{n}$ with $y_{n}=x_{n}-z_{n}$ for each $n \in \mathbb{N}$. Since $y_{n} \rightarrow 0$ as $n \rightarrow \infty$ implies $\gamma\left(\left\{y_{n}\right\}\right)=0$, we have $\gamma\left(\left\{x_{n}\right\}\right) \leq \gamma\left(\left\{y_{n}\right\}\right)+\gamma\left(\left\{z_{n}\right\}\right) \leq \gamma\left(\left\{F x_{n}\right\}\right)$. Since $F$ is countably condensing, the set $\left\{x_{n}\right\}$ is relatively compact. Hence the sequence $\left\{x_{n}\right\}$ has a subsequence $\left\{x_{n_{k}}\right\}$ which converges to some point $x_{0}$ in $X$ and so does $\left\{z_{n_{k}}\right\}$ to the same point. Since $F$ has a closed graph, it follows that $x_{0} \in \partial \Omega$ and $x_{0} \in F x_{0}$, which contradicts the boundary condition.

Now let $G: \bar{\Omega} \rightarrow k c(X)$ be a countably condensing map such that $G x \subset$ $F x+V$ for all $x \in \partial \Omega$. Consider a homotopy $H:[0,1] \times \bar{\Omega} \rightarrow k c(X)$ defined by

$$
H(t, x):=(1-t) F x+t G x \quad \text { for }(t, x) \in[0,1] \times \bar{\Omega} .
$$

Let $C \subset \bar{\Omega}$ be any countable set such that $\gamma(C) \leq \gamma(H([0,1] \times C))$. Since $\gamma(H([0,1] \times C)) \leq \gamma(\operatorname{co}[F(C) \cup G(C)]) \leq \max \{\gamma(F(C)), \gamma(G(C))\}$, we have $\gamma(C) \leq \gamma(F(C))$ or $\gamma(C) \leq \gamma(G(C))$, which implies that $C$ is relatively compact because two maps $F$ and $G$ are countably condensing. Thus, $H$ is countably condensing. Moreover, $x \notin H(t, x)$ for all $(t, x) \in[0,1] \times \partial \Omega$. In fact, if 
$x \in H(t, x)$ for some $(t, x) \in[0,1] \times \partial \Omega$, then $(x-F x) \cap t(G x-F x) \neq \emptyset$ and hence $(x-F x) \cap V \neq \emptyset$, which is a contradiction to the choice of $V$. Therefore, Lemma 1 implies that $\operatorname{deg}(I-G, \Omega, 0)=\operatorname{deg}(I-F, \Omega, 0)$. In particular, for every $y \in V$, if $G_{y} x:=F x+y$ for $x \in \bar{\Omega}$, then $\operatorname{deg}(I-F, \Omega, y)=$ $\operatorname{deg}\left(I-G_{y}, \Omega, 0\right)=\operatorname{deg}(I-F, \Omega, 0)$. This completes the proof.

\section{The locally nonopposite map case}

In this section, we give an invariance of domain theorem for countably condensing multivalued vector fields under the locally nonopposite condition.

Following Ma [7], given an open set $\Omega$ in $X$, a map $G: \Omega \rightarrow k c(X)$ is said to be locally nonopposite if for each $x \in \Omega$ there is a convex symmetric open neighborhood $V$ of the origin 0 in $X$ such that $x+\bar{V} \subset \Omega$ and for all $y \in x+\partial V$ and all $\lambda \geq 0$ we have $\lambda(x-y) \notin G y-G x$.

The following result was first proved by Ma [7, Theorem 19.3] for compact multivalued maps; see [9, Theorem 4.2] for condensing maps.

Theorem 1. Let $\Omega$ be an open subset of a Fréchet space $X$. Suppose that $F: \Omega \rightarrow k c(X)$ is a countably condensing map with respect to $\gamma$ such that $I-F$ is locally nonopposite. Then $(I-F)(\Omega)$ is open.

Proof. We may assume that $\Omega$ is not empty. Let $z \in(I-F)(\Omega)$ and choose a point $x \in \Omega$ so that $z \in x-F x$. Let $V$ be a convex symmetric open neighborhood of 0 in $X$ such that $x+\bar{V} \subset \Omega$ and for all $y \in x+\partial V$ and all $\lambda \geq 0$ we have $\lambda(x-y) \notin(I-F) y-(I-F) x$. Let $H:[0,1] \times(x+\bar{V}) \rightarrow k c(X)$ be a homotopy defined by

$$
H(t, y):=(1-t) x+t(F y+z) \quad \text { for }(t, y) \in[0,1] \times(x+\bar{V}) .
$$

For each countable set $C \subset(x+\bar{V})$, if $\gamma(C) \leq \gamma(H([0,1] \times C))$, then it follows from $\gamma(H([0,1] \times C)) \leq \gamma(\operatorname{co}[(F(C)+z) \cup\{x\}]) \leq \gamma(F(C))$ that $C$ is relatively compact. Thus, $H$ is countably condensing. Moreover, $y \notin H(t, y)$ for all $(t, y) \in[0,1] \times(x+\partial V)$. In fact, if $y \in H(t, y)$ for some $(t, y) \in$ $[0,1] \times(x+\partial V)$, then $x-y \in t(-F y-z+x) \subset t(-F y+F x)$ and hence $(1 / t-1)(x-y) \in(I-F) y-(I-F) x$, which is a contradiction. Therefore, the homotopy invariance and normalization in Lemma 1 imply that

$$
\operatorname{deg}(I-F-z, x+V, 0)=\operatorname{deg}(I-x, x+V, 0)=1 .
$$

Note that $F+z$ is countably condensing on $x+\bar{V}$ and $y \notin F y+z$ for all $y \in x+\partial V$. By Lemma 2, there exists an open neighborhood $W$ of 0 in $X$ such that

$$
\operatorname{deg}(I-F-z, x+V, w)=\operatorname{deg}(I-F-z, x+V, 0) \quad \text { for every } w \in W .
$$

For every $w \in W$, since $\operatorname{deg}(I-F-z, x+V, w) \neq 0$, it follows from the existence property that there exists a point $y_{*} \in x+V$ such that $y_{*} \in F y_{*}+z+w$ and hence $z+w \in(I-F) y_{*}$. Therefore, we obtain that $z+W \subset(I-F)(\Omega)$. This 
means that $z$ is an interior point of $(I-F)(\Omega)$. We conclude that $(I-F)(\Omega)$ is open.

As a consequence of Theorem 1, we obtain the following single-valued version. However, it is not known whether the invariance of domain theorem holds if $f$ is a (countably) condensing map and $I-f$ is (locally) injective; see also Corollary 2 below.

Corollary 1. Let $\Omega$ be an open set in $X$. Suppose that $f: \Omega \rightarrow X$ is a countably condensing single-valued map such that for each $x \in \Omega$ there is a convex symmetric open neighborhood $V$ of 0 in $X$ such that $x+\bar{V} \subset \Omega$ and for all $y \in x+\partial V$ and all $\lambda \geq 0$ we have $(\lambda+1)(x-y) \neq f x-f y$. Then $(I-f)(\Omega)$ is open.

\section{The local boundary map case}

In this section, we establish an invariance of domain theorem for countably $k$-contractive multivalued vector fields under the local boundary condition.

We first give Borsuk's theorem for odd countably condensing multivalued maps which is deduced from the corresponding result for compact maps stated in $[9]$.

Theorem 2. Let $\Omega$ be a symmetric open neighborhood of 0 in a Fréchet space $X$. Let $F: \bar{\Omega} \rightarrow k c(X)$ be an odd countably condensing map such that $x \notin F x$ for all $x \in \partial \Omega$. Then $\operatorname{deg}(I-F, \Omega, 0)$ is odd.

Proof. Suppose that $F: \bar{\Omega} \rightarrow k c(X)$ is an odd countably condensing map such that $x \notin F x$ for all $x \in \partial \Omega$. Then by Proposition 2 , there exists a symmetric compact fundamental set $S$ for $F$ with $0 \in S$ such that $F(\bar{\Omega} \cap S)$ is a subset of $S$. Let $R_{0}: X \rightarrow S$ be any retraction onto $S$ and let $R: X \rightarrow S$ be a map defined by

$$
R x=\frac{1}{2}\left[R_{0} x-R_{0}(-x)\right] \text { for } x \in X .
$$

Then $R$ is an odd retraction onto $S$. In view of Definition 3, we have

$$
\operatorname{deg}(I-F, \Omega, 0)=d_{M}\left(I-F \circ R, R^{-1}(\Omega), 0\right) .
$$

Note that the set $R^{-1}(\Omega)$ is a symmetric open neighborhood of 0 in $X$. The symmetry follows from the fact that $R$ is odd and $\Omega$ is symmetric. Since $F \circ R$ is compact and odd on the closure of $R^{-1}(\Omega)$, Proposition 2.2 of [9] for odd compact multivalued maps implies that $d_{M}\left(I-F \circ R, R^{-1}(\Omega), 0\right)$ is an odd integer. Therefore, we conclude that $\operatorname{deg}(I-F, \Omega, 0)$ is odd. This completes the proof.

In analogy with the single-valued case [6], we need another notion of countably $k$-contractive maps in the strong sense. 
Definition 4. Let $\Omega$ be a nonempty subset of $X, \gamma$ a measure of noncompactness on $X$, and $k$ a nonnegative real number. An upper semicontinuous map $H:[0,1] \times \Omega \rightarrow 2^{X}$ is said to be countably $k$-contractive (with respect to $\gamma$ ) if $\gamma(H([0,1] \times C)) \leq k \gamma(C)$ for each countable set $C \subset \Omega$. Moreover, an upper semicontinuous map $F: \Omega \rightarrow 2^{X}$ is said to be countably $k$-contractive in the strong sense (with respect to $\gamma$ ) if $\gamma(F(\overline{\mathrm{co}} C)) \leq k \gamma(C)$ for each countable set $C$ with $\overline{\mathrm{co}} C \subset \Omega$.

An additional condition $\overline{\mathrm{co}} C \subset \Omega$ may be automatically dropped whenever $\Omega$ is a closed convex subset of $X$. In this case, every countably $k$-contractive map $F: \Omega \rightarrow 2^{X}$ in the strong sense is countably $k$-contractive.

Given an open set $\Omega$ in a Banach space $X$, a map $G: \Omega \rightarrow k c(X)$ is called a local boundary map if for each $x \in \Omega$ there is a convex symmetric bounded open neighborhood $V$ of the origin 0 in $X$ such that $x+\bar{V} \subset \Omega$ and for all $x_{1}, x_{2} \in x+\bar{V}$ with $G x_{1} \cap G x_{2} \neq \emptyset$ we have $x_{1}-x_{2} \notin \partial V$; see [7].

Now we can prove an invariance of domain theorem for countably $k$-contractive multivalued maps in the strong sense. This includes as special cases Theorem 4.1 of [9] for $k$-contractive maps and Theorem 19.2 of [7] for compact maps.

Theorem 3. Let $\Omega$ be an open subset of a Banach space $X$ and $k \in[0,1)$. Let $F: \Omega \rightarrow k c(X)$ be a countably $k$-contractive map in the strong sense with respect to the measure $\gamma$ of noncompactness, where $\gamma=\alpha$ or $\gamma=\beta$ is as in Example 1. If $I-F$ is a local boundary map, then $(I-F)(\Omega)$ is open.

Proof. Without loss of generality we may assume that $\Omega$ is a nonempty proper subset of $X$. Let $z \in(I-F)(\Omega)$ and choose an $x \in \Omega$ so that $z \in x-F x$. Let $V$ be a convex symmetric bounded open neighborhood of 0 in $X$ such that $x+\bar{V} \subset \Omega$ and for all $x_{1}, x_{2} \in x+\bar{V}$ with $(I-F)\left(x_{1}\right) \cap(I-F)\left(x_{2}\right) \neq \emptyset$ we have $x_{1}-x_{2} \notin \partial V$. Let $G: \bar{V} \rightarrow k c(X)$ be a map defined by

$$
G y:=F(x+y)+z-x \text { for } y \in \bar{V} \text {. }
$$

It is easy to see that $G$ is countably $k$-contractive in the strong sense and $0 \in(I-G)(0)$. For all $y_{1}, y_{2} \in \bar{V}$ with $(I-G) y_{1} \cap(I-G) y_{2} \neq \emptyset$, we have $(I-F)\left(x+y_{1}\right) \cap(I-F)\left(x+y_{2}\right) \neq \emptyset$ and hence $y_{1}-y_{2} \notin \partial V$. Consider a homotopy $H:[0,1] \times \bar{V} \rightarrow k c(X)$ given by

$$
H(t, y):=G\left(\frac{1}{1+t} y\right)-G\left(-\frac{t}{1+t} y\right) \quad \text { for }(t, y) \in[0,1] \times \bar{V}
$$

A similar argument used in [6, Lemma 4.2] proves that $H$ is countably $k$ contractive. Notice that $H$ is countably condensing on $[0,1] \times \bar{V}$ because $V$ is bounded and $0 \leq k<1$. Moreover, $y \notin H(t, y)$ for all $(t, y) \in[0,1] \times \partial V$. Indeed, if $y \in H(t, y)$ for some $(t, y) \in[0,1] \times \partial V$, we have

$$
(I-G)\left(\frac{1}{1+t} y\right) \cap(I-G)\left(-\frac{t}{1+t} y\right) \neq \emptyset,
$$


$y_{1}:=1 /(1+t) y \in \bar{V}, y_{2}:=-t /(1+t) y \in \bar{V}$, and $y_{1}-y_{2}=y \in \partial V$, which is a contradiction to the above fact. Now the invariance property of homotopy implies that

$$
\operatorname{deg}(I-G, V, 0)=\operatorname{deg}(I-H(1, \cdot), V, 0) .
$$

In view of Theorem 2 , since $H(1, \cdot)$ is an odd countably condensing map on $\bar{V}$, we have

$$
\operatorname{deg}(I-H(1, \cdot), V, 0) \neq 0 .
$$

Since $G$ is countably condensing on $\bar{V}$ and $y \notin G y$ for all $y \in \partial V$, by Lemma 2 , there is an open neighborhood $W$ of 0 in $X$ such that

$$
\operatorname{deg}(I-G, V, w)=\operatorname{deg}(I-G, V, 0) \neq 0 \quad \text { for every } w \in W .
$$

For every $w \in W$, there exists a $y \in V$ such that $y \in G y+w$ or $z+w \in$ $(I-F)(x+y)$. We conclude that $z$ is an interior point of $(I-F)(\Omega)$. This completes the proof.

We have the following result given in [6, Theorem 4.3]. Recall that a singlevalued map $g: \Omega \rightarrow X$ is said to be locally injective if for each $x \in \Omega$ there exists a neighborhood $V$ of $x$ in $X$ such that the restriction of $g$ to $V$ is injective.

Corollary 2. Let $\Omega$ be an open set in a Banach space $X$ and $k \in[0,1)$. Let $f: \Omega \rightarrow X$ be a countably $k$-contractive map in the strong sense. If $I-f$ is locally injective, then $I-f$ is open.

Proof. Note that every local injection is a local boundary map. For each open subset $\Omega_{0}$ of $\Omega,(I-f)\left(\Omega_{0}\right)$ is open, by Theorem 3. Thus, $I-f$ is open.

Corollary 3. Let $\Omega$ be a domain in a Banach space $X$ and $f: \Omega \rightarrow X$ a completely continuous map. If $I-f$ is locally injective, then $(I-f)(\Omega)$ is a domain in $X$. Here $\Omega$ is a domain if it is open and connected.

Proof. Since $f$ is a completely continuous map, it is obviously countably $k$ contractive in the strong sense with respect to $\gamma$, where $k$ is any nonnegative real number and $\gamma$ is the Kuratowski or Hausdorff measure of noncompactness on $X$, as in Example 1. The conclusion follows from Corollary 2 and the fact that the continuous image of a connected set is connected.

\section{References}

[1] R. R. Akhmerov, M. I. Kamenskii, A. S. Potapov, A. E. Rodkina, and B. N. Sadovskii, Measures of Noncompactness and Condensing Operators, Birkhäuser, Basel, 1992.

[2] K. Deimling, Nonlinear Functional Analysis, Springer, Berlin, 1985.

[3] S. Hahn, Gebietsinvarianz und Eigenwertaussagen für konzentrierende Abbildungen, Comment. Math. Univ. Carolinae 18 (1977), no. 4, 697-713.

[4] M. I. Kamenskii, V. Obukhovskii, and P. Zecca, Condensing Multivalued Maps and Semilinear Differential Inclusions in Banach Spaces, de Gruyter, Berlin, 2001.

[5] I.-S. Kim, On the domain invariance of countably condensing vector fields, J. Math. Anal. Appl. 307 (2005), no. 1, 65-76.

[6] _ The invariance of domain theorem for condensing vector fields, Topol. Methods Nonlinear Anal. 25 (2005), no. 2, 363-373. 
[7] T.-W. Ma, Topological degrees of set-valued compact fields in locally convex spaces, Dissertationes Math. Rozprawy Mat. 92 (1972), 43 pp.

[8] H. Mönch, Boundary value problems for nonlinear ordinary differential equations of second order in Banach spaces, Nonlinear Anal. 4 (1980), no. 5, 985-999.

[9] W. V. Petryshyn and P. M. Fitzpatrick, A degree theory, fixed point theorems, and mapping theorems for multivalued noncompact mappings, Trans. Amer. Math. Soc. 194 (1974), 1-25.

[10] J. Schauder, Invarianz des Gebietes in Funktionalräumen, Studia Math. 1 (1929), 123139.

[11] _ Ü̈ber den Zusammenhang zwischen der Eindeutigkeit und Lösbarkeit partieller Differentialgleichungen zweiter Ordnung vom elliptischen Typus, Math. Ann. 106 (1932), no. 1, 661-721.

[12] M. Väth, Fixed point theorems and fixed point index for countably condensing maps, Topol. Methods Nonlinear Anal. 13 (1999), no. 2, 341-363.

[13] - An axiomatic approach to a coincidence index for noncompact function pairs, Topol. Methods Nonlinear Anal. 16 (2000), no. 2, 307-338.

[14] (2001), no. 1, 223-237.

Department of Mathematics

SUNGKYUNKWAN UNIVERSITY

SuwON 440-746, Korea

E-mail address: iskim@skku.edu 